\title{
Bending rigidity and higher order curvature terms for the hard-sphere fluid near a curved wall
}

\author{
Ignacio Urrutia*† \\ * Departamento de Física de la Materia Condensada, Centro Atómico Constituyentes, \\ CNEA, Av.Gral. Paz 1499, 1650 Pcia. de Buenos Aires, Argentina and \\ $\dagger$ CONICET.
}

\begin{abstract}
In this work I derive analytic expressions for the curvature dependent fluid-substrate surface tension of a hard sphere fluid on a hard curved wall. In a first step, the curvature thermodynamic properties are found as truncated power series in the activity in terms of the known second and third order cluster integral of the hard-sphere fluid near spherical and cylindrical walls. These results are then expressed as packing fraction power series and transformed to different reference regions which is equivalent to consider different positions of the dividing surface. All the obtained series expansions are rigorous results. Based on the truncated series it is shown that the bending rigidity of the system is non-null and that higher order terms in the curvature also exist. In a second step, approximate analytic expressions for the surface tension, the Tolman length, the bending rigidity and the Gaussian rigidity as functions of the packing fraction are found by considering the known terms of the series expansion complemented with a simple fitting approach. It is found that the obtained formulas accurately describe the curvature thermodynamic properties of the system, further, they are more accurate than any of the previously published expressions.
\end{abstract}

\section{INTRODUCTION}

The relation between the thermodynamic properties of a confined fluid and the shape of the vessel where it is confined has become a topic of current interest. Recently, the inhomogeneous fluid cluster expansion was revisited and applied to the hard sphere (HS) fluid near a hard planar-wall [1]. Moreover, the HS system near spherical and cylindrical hard walls was studied focusing on the analysis of the curvature dependent fluid-wall surface tension using both, molecular dynamics and the density functional theory [2-4].

The studies of the local dependence of the surface tension or surface free-energy on the curvature (for membranes, for two fluid-phases and also for fluid/wall, systems) rely in one of the two following different expressions. The first one, derived by Helfrich is [5]

$$
\gamma(J, K)=\gamma-\delta \gamma J+\frac{k}{2} J^{2}+\bar{k} K+\ldots,
$$

where the truncation to order $K$ is frequently assumed. The second one, proposed by König et al. and based on the Hadwiger Theorem [6] is

$$
\gamma(J, K)=\gamma-\delta \gamma J+\bar{k} K
$$

In Eqs. (1) 2) $J=R_{1}^{-1}+R_{2}^{-1}$ is the total curvature, $K=R_{1}^{-1} R_{2}^{-1}$ is the Gaussian curvature, and $R_{1}, R_{2}$ are the local principal radius of the surface. The fluid-wall surface tension (or surface free-energy) for the case of a planar wall is indicated by $\gamma$, while the curvature coefficients are: $\delta$ the Tolman's length, $k$ the bending rigidity and $\bar{k}$ the Gaussian-curvature rigidity. Both expressions were successfully utilized to describe, on approximate grounds, the properties of interfaces. Furthermore, it was conjectured that Eq. (2) is complete for the HS fluid in contact with hard curved walls [7 9$]$.
The analysis of the curvature dependent surface tension is particularly simple in the context of constant curvature surfaces like cylindrical and spherical wall/fluid interfaces. In the last decade, different studies about HS under these geometrical constraints were dedicated to evaluate the bending rigidity, $k$. On the framework of fundamental measure theory of Rosenfeld (FMT), and based on the numerical analysis of the free energy it were found bending rigidity values compatible with $k=0$ [ 9]. On the other hand, on the framework of a similar FMT it was derived an integral expression for $k$ providing $k \neq 0$ and suggesting that Eq. (2) is not complete for this system. A second evidence supporting $k \neq 0$ result from the re-examination of molecular dynamics (MD) results [3, 4]. Unfortunately, the smallness of the obtained maximum value for $k$ at moderate low packing fraction $\eta$ (smaller than 0.3) $k(\eta=0.2)=0.000742$ using FMT and $k(\eta=0.25)=0.001 \pm 0.0008$ using MD, makes necessary to take them with caution (here $k$ is in $k_{\mathrm{B}} T$ units, being $T$ the temperature and $k_{\mathrm{B}}$ Boltzmann constant). On one hand, because the method used to extract the curvature terms from MD depends on a fit which is very sensible on the adopted procedure. On the other hand, because FMT is an approximate theory that produce reliable results but non-necessarily to this higher degree of accuracy.

In this work I study the higher order curvature dependence of the surface tension for the HS fluid confined by a spherical and cylindrical hard walls. The curvaturethermodynamic properties are obtained as power series in the activity and packing fraction and its coefficients to order two (for $k$ and $\bar{k}$ ) or to order three (for $\delta \gamma, 2 k+\bar{k}$, and the terms of order $R^{-3}$ in Eq. (1) ) are obtained exactly. An important finding presented here is that $k \neq 0$ on exact grounds and thus Eq. (2) is an approximated expression for the studied system. The effect that the change of the reference region produces on the system 
properties is revisited. The low order series expansions of curvature-thermodynamic properties are also found by adopting a dividing surface typical of the scaled particle theory. Using the known terms of the series expansions complemented with the fitting of available data simple and accurate analytic expressions are presented for $\delta \gamma$, $2 k+\bar{k}$ and for the first time for $k$ and $\bar{k}$. These expressions transformed to different reference regions are verified by comparing with available data and it is concluded that they are the most accurate description for the analyzed properties developed at present.

\section{EXPANSION IN THE ACTIVITY}

The grand potential of a fluid of point-particles confined in a region $\mathcal{A}$ by a hard wall (not necessarily a planar wall) is

$$
\beta \Omega=-\sum_{i \geq 1} \frac{\tau_{i}}{i !} z^{i},
$$

with $\tau_{i}$ the $i$-particles cluster integral of the fluid-in- $\mathcal{A}$ system, $\beta=1 / k_{\mathrm{B}} T$ its inverse temperature and $z=$ $\Lambda^{-3} \exp (-\beta \mu)$ its activity. Other magnitudes are: $\mu$ the chemical potential, $\Lambda$ the de Broglie thermal length and $\beta \Omega=-\ln \Xi$, being $\Xi$ the grand canonical partition function. For both, spherical and cylindrical hard walls with a large enough radii $R$, the cluster integrals can be written as

$$
\frac{\tau_{i}}{i !}=b_{i} V-a_{i} A+c_{i, 1} \frac{A}{R}+c_{i, 2} \frac{A}{R^{2}}+c_{i, 3} \frac{A}{R^{3}}+\ldots,
$$

where $V$ is the volume of the system, $A$ is its surface area and $R$ its radius. At constant temperature the coefficients $b_{i}, a_{i}$ and $c_{i, j}$ with $j \geq 1$, are constant. Coefficients $b_{i}$ and $a_{i}$ are universal and correspond to the bulk cluster integrals [10] and to the planar-wall surface cluster integrals [11, 12], respectively. On the other hand, $c_{i, j}$ may depend on the shape of the region where the fluid is confined. Naturally, if the wall is planar $c_{i, j}=0$ for all $j$. Note that $V, A, R, b_{i}, a_{i}$ and $c_{i, j}$ refer to a given reference region $\mathcal{B}$ that follows the spherical/cylindrical symmetry of the one-body distribution function. Once $\mathcal{B}$ is fixed (through its radius $R=R(\mathcal{B})$ ) the description given in Eq. (4) is unique. Eqs. (3, 4) suggest that

$$
\Omega=-P V+\gamma A+C_{1} \frac{A}{R}+C_{2} \frac{A}{R^{2}}+C_{3} \frac{A}{R^{3}}+\ldots,
$$

or equivalently

$$
\begin{aligned}
\Omega & =-P V+\gamma(R) A, \\
\gamma(R) & =\gamma+C_{1} / R+C_{2} / R^{2}+C_{3} / R^{3}+\ldots,
\end{aligned}
$$

with $P=\sum_{i \geq 1} b_{i} z^{i}$ the pressure of the bulk system (at the same $z$ and $T$ ) and

$$
\gamma=\sum_{i \geq 1} a_{i} z^{i}, C_{j}=-\sum_{i \geq 1} c_{i, j} z^{i},
$$

$$
\gamma(R)=\sum_{i \geq 1}\left(a_{i}-\sum_{j \geq 1} c_{i, j} R^{-j}\right) z^{i} .
$$

Here, $\gamma(R)$ is the curvature dependent fluid-wall surface tension and the functions $C_{j}$ are thermodynamic curvature coefficients. The fluid-wall hard potential induces the formation of a surface with radius $R_{\mathrm{d}}$ where the one body density distribution, $\rho(\mathbf{r})$, drops discontinuously to zero. The pressure on this zero-density surface, $P_{\mathrm{o}}$, is given by the ideal-gas-like relation $P_{\mathrm{o}} / k_{\mathrm{B}} T=\rho\left(R_{\mathrm{d}}\right)$, which is known as the wall or contact theorem. Given that $P_{\mathrm{o}}=-\frac{d R}{d V} \frac{\partial}{\partial R} \Omega$ one found

$$
\begin{aligned}
& P_{\mathrm{o}}=P+\gamma \frac{2}{R}+C_{1} \frac{1}{R^{2}}-C_{3} \frac{1}{R^{4}}+\ldots,(\text { sph }) \\
& P_{\mathrm{o}}=P+\gamma \frac{1}{R}-C_{2} \frac{1}{R^{3}}-C_{3} \frac{2}{R^{4}}+\ldots,(\text { cyl })
\end{aligned}
$$

where $P=-\partial \Omega / \partial V, \gamma=\partial \Omega / \partial A, C_{j}=\partial \Omega / \partial\left(A R^{-j}\right)$ and it was assumed that $R-R_{\mathrm{d}}$ is a constant length. The mean number of particles $N=-z \frac{\partial}{\partial z} \frac{\Omega}{k T}=\sum_{i \geq 1} i \frac{\tau_{i}}{i !} z^{i}$ can also be decomposed as Eqs. (5.9) resulting expressions like $N=\rho V+\Gamma A+\Gamma^{(1)} A / R+\ldots$ and $N=$ $\rho V+\Gamma(R) A$ where the density of the bulk system at the same $z$ and $T$ is

$$
\rho=\sum_{i \geq 1} i b_{i} z^{i}
$$

$\Gamma$ is the adsorption on a planar wall, $\Gamma^{(1)}$ is the first pure curvature adsorption, etc. In fact, the same method apply to higher order derivatives too, for example to the fluctuation in the number of particles $\left\langle N^{2}\right\rangle-N^{2}=$ $z \frac{\partial N}{\partial z}=\sum_{i \geq 1} i^{2} \frac{\tau_{i}}{i !} z^{i}$.

The Helfrich expansion given in Eq. (11) was originally derived for closed vesicles [5]. Vesicles are symmetric in the sense that substance inside and outside are the same, therefore in this case the sign of $J$ is fixed and can be chosen by convention. To apply Eq. (11) to the unsymmetrical system of a fluid in contact with a constant curvature hard wall I adopted the usual convention taking the curvature radius $R_{l}$ as positive for the fluid outside of the spherical or cylindrical body. Therefore, in this work Eq. (11) reduces to

$$
\begin{aligned}
& \gamma(R)=\gamma-2 \frac{\delta \gamma}{R}+\frac{2 k+\bar{k}}{R^{2}}+\ldots,(\text { sph }) \\
& \gamma(R)=\gamma-\frac{\delta \gamma}{R}+\frac{k}{2 R^{2}}+\ldots,(\text { cyl })
\end{aligned}
$$

where higher order terms like $C_{3(s p h)}$ and $C_{3(c y l)}$ are included. These relations can be compared with Eq. (77) to obtain

$$
\begin{gathered}
\delta \gamma_{(s p h)}=-\frac{1}{2} C_{1(s p h)}, c k_{(s p h)}=C_{2(s p h)}, \\
\delta \gamma_{(c y l)}=-C_{1(c y l)}, k_{(c y l)}=2 C_{2(c y l)},
\end{gathered}
$$

where it was introduced the combined rigidity in short notation $c k=2 k+\bar{k}$. Based on Helfrich's expression, 


\begin{tabular}{|c|c|c|c|c|}
\hline$i$ & 2 & 3 & 4 VI & 5VI \\
\hline \hline$b_{i}$ & $-2 \pi / 3$ & $3 \pi^{2} / 4$ & $-32.6506 \ldots$ & $162.9498822(5)$ \\
\hline$a_{i}$ & $-\pi / 8$ & $\frac{137}{560} \pi^{2}$ & $-14.3871(6)$ & $88.053(10)$ \\
\hline
\end{tabular}

Table I. Bulk and surface (planar-wall) coefficients of the cluster integral $\tau_{i}$ up to $i=5 . b_{i}$ and $a_{i}$ are expressed in units of $\sigma^{3}$ and $\sigma^{4}$, respectively.

\begin{tabular}{|c|c|c|c|}
\hline$i$ & $2(\mathrm{sph})$ & $3(\mathrm{sph})$ & $2(\mathrm{cyl})$ \\
\hline \hline$c_{i, 1}$ & 0 & $-\frac{\pi}{768}(9 \sqrt{3}+16 \pi)$ & 0 \\
\hline$c_{i, 2}$ & $-\frac{\pi}{144}$ & $\frac{781}{36288} \pi^{2}$ & $-\frac{\pi}{384}$ \\
\hline$c_{i, 3}$ & 0 & $-\frac{\pi}{1280 \sqrt{3}}$ & 0 \\
\hline
\end{tabular}

Table II. Curvature coefficients of the cluster integral $\tau_{2}$ and $\tau_{3}$. Known terms for spherical and cylindrical hard wall up to $j=3$. $c_{i, j}$ has units of $\sigma^{4+j}$.

none of the magnitudes $\gamma, \delta, k$ and $\bar{k}$ depend on the geometry and thus (sph) and (cyl) labels should be unnecessary.

\section{THE HS FLUID}

For the fluid of HS with hard repulsion distance $\sigma$ (from here on when becomes convenient $\sigma$ will be considered $\sigma=1$ for simplicity) in contact with a hard sphere wall $\tau_{2}$ and $\tau_{3}$ were evaluated in Refs. [13, 14]. In addition, for the case of a hard cylindrical wall the expression of $\tau_{2}$ was found in Ref. [15]. Instead, the cluster integrals $\tau_{i}$ for $i>3$ are only partially known from the values of $b_{i}$ and $a_{i}$ [16] [17]. The coefficients $b_{i}$ and $a_{i}$ for $i=2,3,4,5$ are listed in Table [. Table \summarizes the known curvature coefficients of $\tau_{i}$ for the spherical and cylindrical hard-walls up to $j=3$. These coefficients correspond to a choice of the reference region $(\mathrm{RR}) \mathcal{B}$ that coincides with the available region for the center of each HS, $\mathcal{A}$, with $\rho(\mathbf{r} \notin \mathcal{A})=0$. Thus, under this density-based RR (d-RR) results $R=R_{\mathrm{d}}$, which determines the position of the surface of tension, being $A=A_{\mathrm{d}}=4 \pi R_{\mathrm{d}}$ and $V=V_{\mathrm{d}}=4 \pi R_{\mathrm{d}} / 3$. d-RR makes easy the evaluation of $\tau_{i}$ (eg. in d-RR $\tau_{1}=V$ ). Although using Eqs. (8, 15) it is possible to explicitly write the curvature-thermodynamic properties as series expansion in powers of $z$, it is customary to show the results as function of the packing fraction $\eta=\rho \sigma^{3} \pi / 6$. Therefore, it is necessary to invert the series of Eq. (12) to find the series of $z(\eta)$, and then compose series to obtain the series expansion of each property in powers of $\eta$. Pressure and surface tension series reproduce the well known virial series results, eg. $\beta \gamma=-\frac{9 \eta^{2}}{2 \pi}\left(1+\frac{149}{35} \eta\right)+O\left(\eta^{3}\right)$. For the spherical wall the exact series expansions of the curvature-thermodynamic properties gives

$$
\beta \delta \gamma_{(s p h)}=-\frac{9}{64 \pi^{2}}(9 \sqrt{3}+16 \pi) \eta^{3}+O\left(\eta^{4}\right)
$$

$$
\beta c k_{(s p h)}=\frac{\eta^{2}}{4 \pi}-\frac{109 \eta^{3}}{168 \pi}+O\left(\eta^{4}\right),
$$

and $\beta C_{3(s p h)}=\frac{9 \sqrt{3}}{160 \pi^{2}} \eta^{3}+O\left(\eta^{4}\right)$. On the other hand, for the cylindrical wall the exact series expansions are

$$
\beta \delta \gamma_{(c y l)}=O\left(\eta^{3}\right), \quad \beta k_{(c y l)}=\frac{3 \eta^{2}}{16 \pi}+O\left(\eta^{3}\right),
$$

and $\beta C_{3(c y l)}=O\left(\eta^{3}\right)$. Note that $\delta \gamma_{(s p h)}$ and $\delta \gamma_{(c y l)}$ are consistent with the Helfrich's expansion at least up to the highest order for which both are known, i.e. $\delta \gamma_{(s p h)}=\delta \gamma_{(c y l)}=\delta \gamma$ up to $O\left(\eta^{3}\right)$. Now, following Helfrich's expansion one found

$$
\begin{gathered}
\beta \delta \gamma=-\frac{9}{64 \pi^{2}}(9 \sqrt{3}+16 \pi) \eta^{3}+O\left(\eta^{4}\right), \quad(\mathrm{d}-\mathrm{RR}) \\
\beta c k=\frac{\eta^{2}}{4 \pi}-\frac{109 \eta^{3}}{168 \pi}+O\left(\eta^{4}\right), \quad(\mathrm{d}-\mathrm{RR})
\end{gathered}
$$

while the rigidity coefficients are

$$
\beta k=\frac{3 \eta^{2}}{16 \pi}+O\left(\eta^{3}\right), \beta \bar{k}=-\frac{\eta^{2}}{8 \pi}+O\left(\eta^{3}\right) \cdot(\mathrm{d}-\mathrm{RR})
$$

Moreover, $c_{i, 1(c y l)}=c_{i, 1(s p h)} / 2$ showing that the unknown coefficient $c_{3,1(c y l)}$ (see Tab. III) is indeed $c_{3,1(c y l)}=-\frac{\pi}{384}(9 \sqrt{3}+16 \pi)$.

\section{DIFFERENT REFERENCE REGIONS}

There are at least two different RR adopted in the literature. In the context of FMT it is usual refer to the d-RR with radius $R=R_{\mathrm{d}}$ already discussed in Sec. III [4]. On the other hand, in the scaled particle theory the focus is usually in the empty region (e-RR) that has a shifted radius of $R=R_{\mathrm{e}}=R_{\mathrm{d}}-1 / 2$ (from here on magnitudes referred to e-RR will be labeled with e). No matter the reference adopted $\tau_{i}$ and $\Omega$ remains invariant because the system remains unmodified. To discuss the change of reference it is convenient to write the Eq. (4) in matrix notation as

$$
\tau_{i} / i !,=\left(\mathbf{b}_{i}\right)_{r} \mathbf{M}_{r},
$$

where up to $O\left(A R^{-4}\right)$ the vector of coefficients is $\mathbf{b}_{i}=$ $\left(b_{i},-a_{i}, c_{i, 1}, c_{i, 2}, c_{i, 3}\right)$ and the column-matrix of measures is $\mathbf{M}=\left(V, A, A R^{-1}, A R^{-2}, A R^{-3}\right)$. In fact, both $\mathbf{b}_{i}$ and $\mathbf{M}$ are relative to the adopted RR, and thus, I introduced the generic label $r$ to make it explicit. In Sec. III. the system was described on the basis of measures $\mathbf{M}_{\mathrm{d}}=\left(V_{\mathrm{d}}, A_{\mathrm{d}}, A_{\mathrm{d}} R_{\mathrm{d}}^{-1}, A_{\mathrm{d}} R_{\mathrm{d}}^{-2}, A_{\mathrm{d}} R_{\mathrm{d}}^{-3}\right)$ and the corresponding vector of coefficients $\left(\mathbf{b}_{i}\right)_{\mathrm{d}}$. Measures taken with different $\mathrm{RR}$ are related by a linear transformation while the inverse transformation relates the corresponding coefficients. Here, the procedure is described for a RR with shifted radius $R=R_{u}=R_{\mathrm{d}}-u$ that corresponds to 
measures $\mathbf{M}_{u}=\left(V_{u}, A_{u}, A_{u} R_{u}^{-1}, A_{u} R_{u}^{-2}, A_{u} R_{u}^{-3}\right)$ with the obvious definition for the volume and surface area of the sphere and the cylinder. In matrix form one found

$$
\tau_{i} / i !=\left(\mathbf{b}_{i}\right)_{\mathrm{d}} Y^{-1} Y \mathbf{M}_{\mathrm{d}}=\left(\mathbf{b}_{i}\right)_{u} \mathbf{M}_{u}
$$

where $\mathbf{M}_{u}=Y \mathbf{M}_{\mathrm{d}}$ and $\left(\mathbf{b}_{i}\right)_{u}=\left(\mathbf{b}_{i}\right)_{\mathrm{d}} Y^{-1}$. To build the matrix $Y$, each measure in the $u$-RR frame is written as a linear function of the measures in the d-RR, for example, $V_{u}=V_{\mathrm{d}}+u A_{\mathrm{d}}-u^{2} A_{\mathrm{d}} R^{-1}+\frac{u^{3}}{3} A_{\mathrm{d}} R^{-2}$. Therefore, $Y_{(s p h)}$ and $Y_{(c y l)}$ are given by

$$
\left(\begin{array}{ccccc}
1 & u & -u^{2} & u^{3} / 3 & 0 \\
0 & 1 & -2 u & u^{2} & 0 \\
0 & 0 & 1 & -u & 0 \\
0 & 0 & 0 & 1 & 0 \\
0 & 0 & 0 & 0 & 1
\end{array}\right) \text { and }\left(\begin{array}{ccccc}
1 & u & -u^{2} / 2 & 0 & 0 \\
0 & 1 & -u & 0 & 0 \\
0 & 0 & 1 & 0 & 0 \\
0 & 0 & 0 & 1 & u \\
0 & 0 & 0 & 0 & 1
\end{array}\right)
$$

respectively (where terms of order $O\left(A_{\mathrm{d}} R^{-4}\right)$ were depreciated). The relation between $\left(\mathbf{b}_{i}\right)_{u}$ and $\left(\mathbf{b}_{i}\right)_{\mathrm{d}}$ directly implies the relation for the extensive-like properties: $\left(-P, \gamma, C_{1}, C_{2}, C_{3}\right)_{u}=\left(-P, \gamma, C_{1}, C_{2}, C_{3}\right)_{\mathrm{d}} Y^{-1}$. In particular, taking $u=1 / 2$ it is possible to derive the properties in the e-RR for both, the sphere and the cylinder, cases. Thus, adopting the Helfrich expansion it is found up to $O\left(\eta^{4}\right)$

$$
\begin{array}{ll}
\beta \delta \gamma=-\frac{3 \eta}{4 \pi}\left[1+\eta+\left(\frac{8}{35}+\frac{27 \sqrt{3}}{16 \pi}\right) \eta^{2}\right], & (\mathrm{e}-\mathrm{RR}) \\
\beta c k=\frac{\eta}{4 \pi}\left[1+\frac{\eta}{2}+\left(\frac{81 \sqrt{3}}{16 \pi}-\frac{289}{105}\right) \eta^{2}\right], & (\mathrm{e}-\mathrm{RR})
\end{array}
$$

while the rigidity constants up to $O\left(\eta^{3}\right)$ take the form

$$
\beta k=\frac{3 \eta^{2}}{16 \pi}, \quad \beta \bar{k}=\frac{\eta}{4 \pi}(1-\eta) . \quad(\mathrm{e}-\mathrm{RR})
$$

Besides, the next terms in higher order of $R^{-1}$ are $\beta C_{3(s p h)}=\frac{9 \sqrt{3} \eta^{3}}{160 \pi^{2}}+O\left(\eta^{4}\right)$ and $\beta C_{3(c y l)}=\frac{3 \eta^{2}}{64 \pi}+O\left(\eta^{3}\right)$. It is noteworthy that $\beta k\left(\beta C_{3(s p h)}\right)$ is independent of the chosen radius of the $\mathrm{RR}$ to any order in $\eta$ because the fourth (fifth) column of $Y_{(c y l)}\left(Y_{(s p h)}\right)$ is equal to the respective column of the identity matrix.

\section{DEPENDENCE OF THE EOS ON $\eta$}

Expressions for $\gamma, \delta \gamma$ and $c k$ as functions of $\eta$ in e$\mathrm{RR}$ were obtained previously by Reiss et al. using the scaled particle theory (SPT) [18] and more recently by Hansen-Goss and Roth [19] by combining an FMT approach known as WBII with Eq. (2). Both sets of expressions were only utilized in e-RR while their accuracy under the adoption of a different RR was not verified. Yet, here I present a third set of expressions for the $\eta$ dependence of $\gamma, \delta \gamma$ and $c k$ based on the known firsts

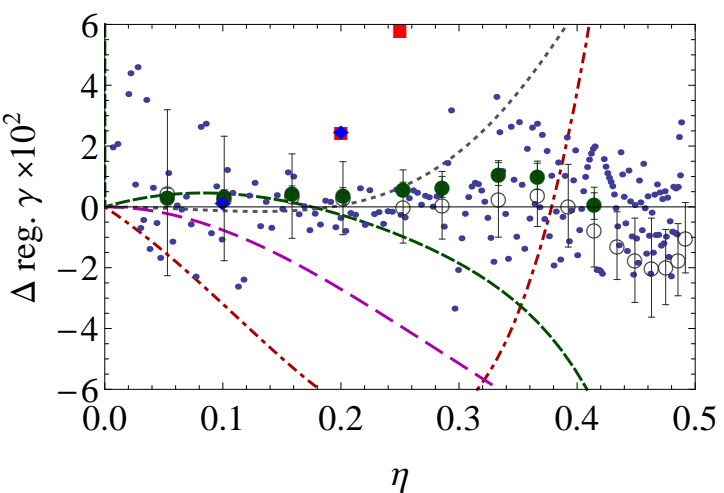

Figure 1. (Color online) Difference between several results for the surface tension and the purposed analytic expression Eq. (28) (in units of $k_{\mathrm{B}} T / \sigma^{2}$ ) by adopting the d-RR. Plotted is the regularized magnitude $\gamma$ (see the text). Circles (open and closed) are MD results from Refs. 2, 3], squares (righted in red and slanted in blue) correspond to FMT results from Refs. [4, 7] while points are MonteCarlo results. Dot-dashed (red) line is the SPT result, dashed (green) line is the WBII result, long-dashed (magenta) line corresponds to the Henderson and Plischke [21] expression while dotted (black) line was obtained by Yang et al. [1].

terms of their power series in $\eta$. Each of these three sets of functions, complemented with the bulk pressure EOS to built $(P, \gamma, \delta \gamma, c k)$, is compared with FMT and Molecular Dynamics (MD) results to evaluate its performance in both d-RR and e-RR. Furthermore, under the same approach I found for the first time expressions for $k$ and $\bar{k}$ as functions of $\eta$.

In the present proposal the functional dependence on $\eta$ for each property will be obtained using the known exact low order series terms and including one fitting parameter. To ensure that the thermodynamic properties are well described regardless the adopted $R R$ the fitting is done in the framework of d-RR and after transformed to the e-RR. In making the transformation between different RRs small inaccuracies in the equations of state may magnify. Therefore, to transform consistently one must ensure that accurately describe the pressure and the surface tension. For $\beta P(\eta)$ one can adopt the very accurate Kolafa-Malijevsky low density (KMlow) EOS[20], however, I verified that the use of the Carnahan-Starling (CS) EOS instead of KM-low introduces very small changes and thus the simple and quasiexact $\operatorname{CS} \beta P(\eta) / \rho=\left(1+\eta+\eta^{2}-\eta^{3}\right) /(1-\eta)^{3}$ is utilized. On the other hand, there is not a sufficiently accurate EOS for the surface tension at present. Here, following the CS rational expression for $P$ I propose,

$$
\beta \gamma=-\frac{9 \eta^{2}}{2 \pi} \frac{1+\frac{44}{35} \eta+\frac{1}{38} \eta^{2}-3(1-\eta) \eta^{3}}{(1-\eta)^{3}}
$$

where the parameter is obtained by fitting and then transformed to the fraction $\frac{1}{38}$. In Fig. 1 it is plotted the regularized difference between the fluid-wall surface 


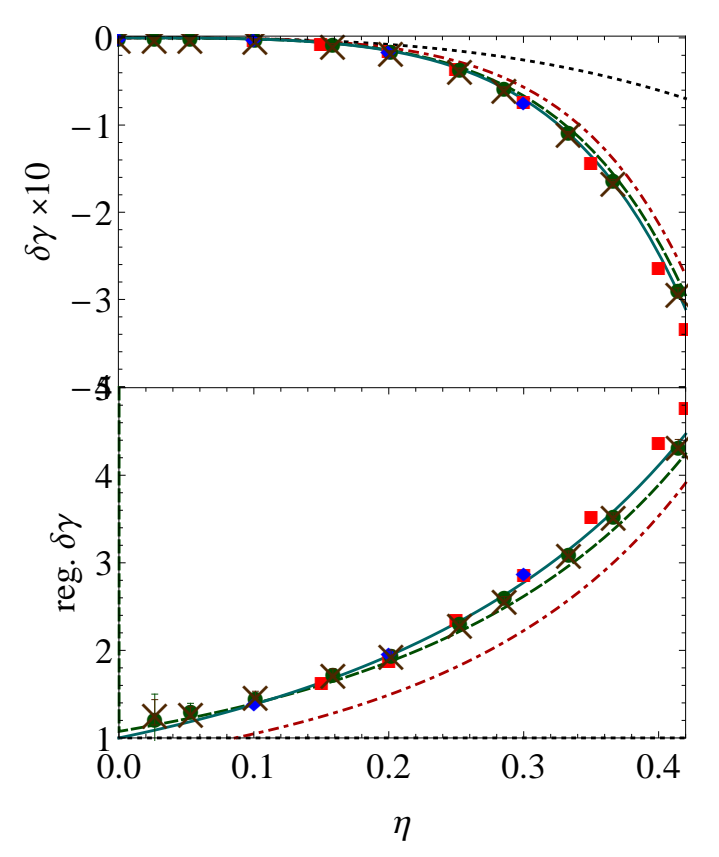

Figure 2. (a) Tolman length in d-RR (in units of $k_{\mathrm{B}} T / \sigma$ ). (b) Regularized Tolman length. Squares are FMT results from Bryk et al. [7] (rigthed-squares in red) and from Blokhuis [4] (slanted-squares in blue), circles (green) and crosses are MD results by Laird et al. [3]. Continuous line (ligthblue) is the proposed expression, dot-dashed (red) line is the SPT result, dashed (green) line is the WBII result while dotted line is the exact series expansion truncated at the last known term.

tension $\gamma$ taken from different sources and that given by Eq. (28), where the regularized version of a magnitude $X$ (reg. $X)$ is obtained by dividing $X$ with the first term of its power series in $\eta$ (eg. reg. $\gamma=\gamma /\left(-9 \eta^{2} / 2 \pi\right)$ ). The plotted symbols are: open and closed circles for Molecular dynamic data (MD) taken from Refs. [2, 3], respectively, squares (righted in red and slanted in blue) correspond to density functional FMT results from Refs. [4, 7] and points are MonteCarlo results [22]. It is evident that Eq. (28) accurately describes the data while other expressions for $\gamma(\eta)$ (which are shown as different curves) deviate at $\eta \gtrsim 0.25$. For the curvature-thermodynamic properties I also utilized fitting functions that combine rational and polynomial forms with one free-parameter. They are

$$
\begin{aligned}
& \beta \delta \gamma=-\left(\frac{9}{4 \pi}+\frac{81 \sqrt{3}}{64 \pi^{2}}\right) \frac{\eta^{3}(1+1.2 \eta)}{(1-\eta)^{2}}, \quad(\mathrm{~d}-\mathrm{RR}) \\
& \beta c k=\frac{\eta^{2}}{4 \pi} \frac{1-\frac{193 \eta}{42}}{(1-\eta)^{2}}-0.54 \eta^{4}(1+3 \eta), \quad(\mathrm{d}-\mathrm{RR})
\end{aligned}
$$

where 1.2 and -0.54 are fitting coefficients. In Fig. 2 (a) the different symbols show the same behavior with increasing $\eta$, which is well described by WBII and fitted lines, while SPT curve is slightly above. Reg. $\delta \gamma$ is presented in Fig. 22 (b). There, the FMT data is slightly separated from MD showing some degree of dis-

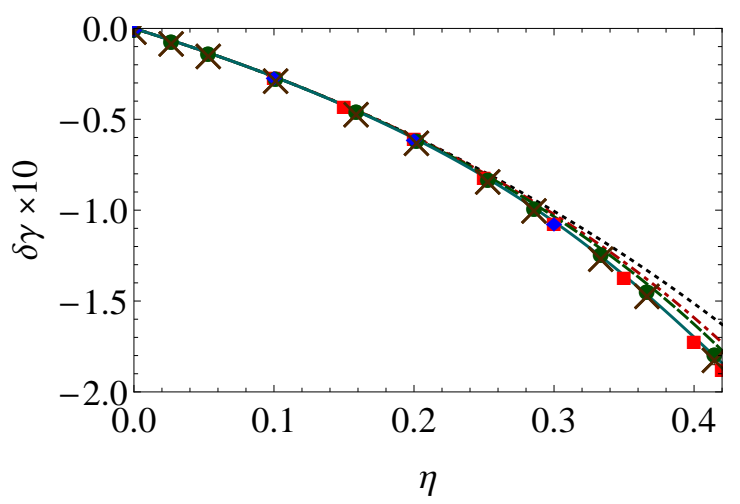

Figure 3. Tolman length times the surface tension in e-RR (in units of $\left.k_{\mathrm{B}} T / \sigma\right)$. Lines and symbols were described in Fig. 2.

crepancy between them, and also, SPT curve does not reproduce MD and FMT data with sufficient accuracy. On the other hand, WBII curve correctly describes the data being the fitted curve the most accurate. Given that the first non-null coefficient of $\delta \gamma$ as a power series in $\eta$ is wrong for the SPT formulae in d-RR its failure is not surprising. It is interesting to compare the FMT results found by Blokhuis and WBII curve, based on slightly different FMT approaches. At $\eta=0.3$ the difference between both values of reg. $\delta \gamma$ is $\sim 0.15$. This small difference is relevant because both methods are accurate in the sense that both involve negligible absolute errors and thus this disagreement is produced by minimal differences in the involved approximations. In this and next figures I include results from a third order polynomial fit in the reciprocal radius of the MD results [3] for the curvature dependent surface tension of spherical and cylindrical hard walls. These results, plotted using crosses, were obtained by first writing the $\gamma(R)$ data in each $\mathrm{d}$ $\mathrm{RR}$ and e-RR, and then fitting it. The Fig. 3 shows that Eq. (29) (found in d-RR and then transformed to e-dd) describes better than the other functional expressions the behavior of the plotted symbols, and also, that the series in $\eta$ truncated to order $\eta^{3}$ follows the symbols in e-RR much better than in d-RR. This last advantage of e-RR with respect to d-RR will be confirmed in the next figures. The results for the combined curvature term $c k$ are

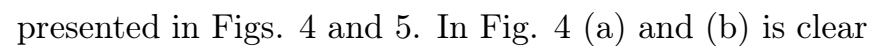
that the best description of the behavior of MD and FMT data is done by Eq. (30) being the WBII curve slightly worse while the worst of the three is that of SPT. Fig. 5 shows that in e-RR the SPT and WBII produce nearly identical results which deviate from the symbols at large $\eta$. Instead, the order-three series truncation given by Eq. (26) accurately describes the symbols. Again, one verify that in e-RR the best curve is provided by the present proposal. The difference between Blokhuis FMT results and WBII curve in Figs. 4 (a), (b) and 5 are apparent and grows with increasing packing fraction. On general grounds, WBII describes the behavior of $\delta \gamma$ and $c k$ in both $\mathrm{d}-\mathrm{RR}$ and e-RR better than SPT while the pro- 


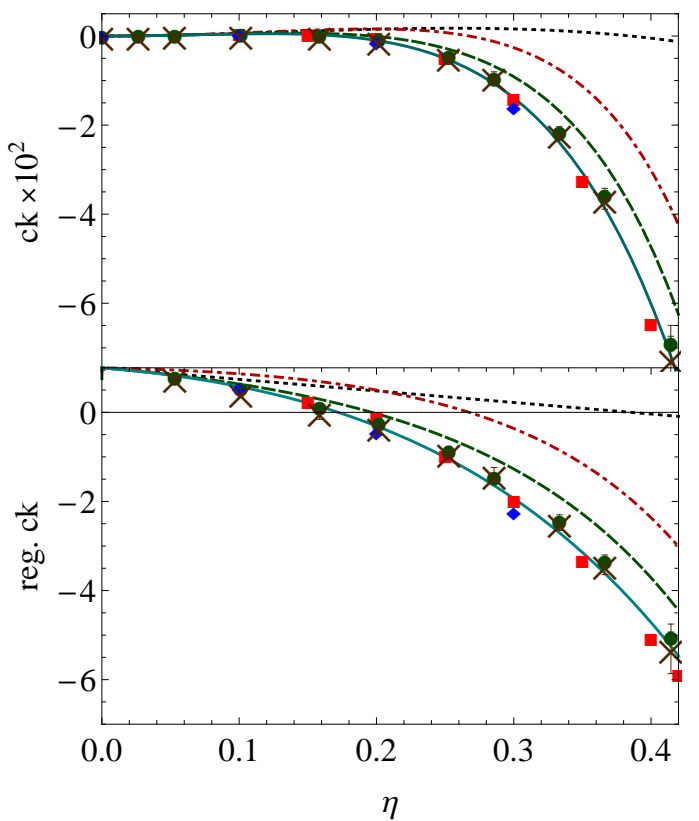

Figure 4. (a) Combined curvature rigidity $c k=2 k+\bar{k}$ in d$\mathrm{RR}$ (in units of $k_{\mathrm{B}} T$ ). (b) Magnitude plotted in (a) but in its regularized form. Lines and symbols were described in Fig. 2

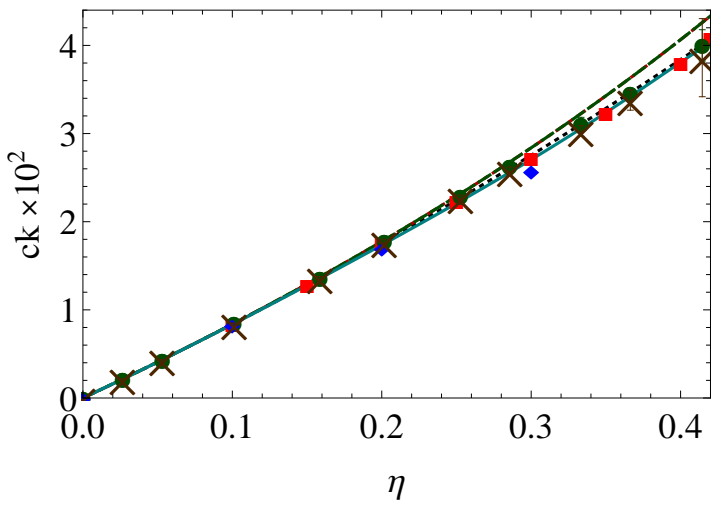

Figure 5. Combined curvature rigidity in e-RR (in units of $\left.k_{\mathrm{B}} T\right)$. Lines and symbols were described in Fig. 2

posed expressions make it still better. In addition, the observed difference between FMT data and WBII suggest that one of them or both FMT-based results might be turning non-confidence.

Expressions for the bending rigidity $k$ and the Gaussian-curvature rigidity $\bar{k}$ were, up to my best knowledge, never presented in the literature. Now, for the bending rigidity the simple dependence

$$
\beta k=\frac{3 \eta^{2}}{16 \pi}(1-3 \eta), \quad(\mathrm{e}+\mathrm{d}-\mathrm{RR})
$$

is proposed based on Eqs. (21, 27) and the overall analysis of the data of regularized $k$. There the adjusted coefficient is -3 . The Fig. [6] shows results for $k$ in both

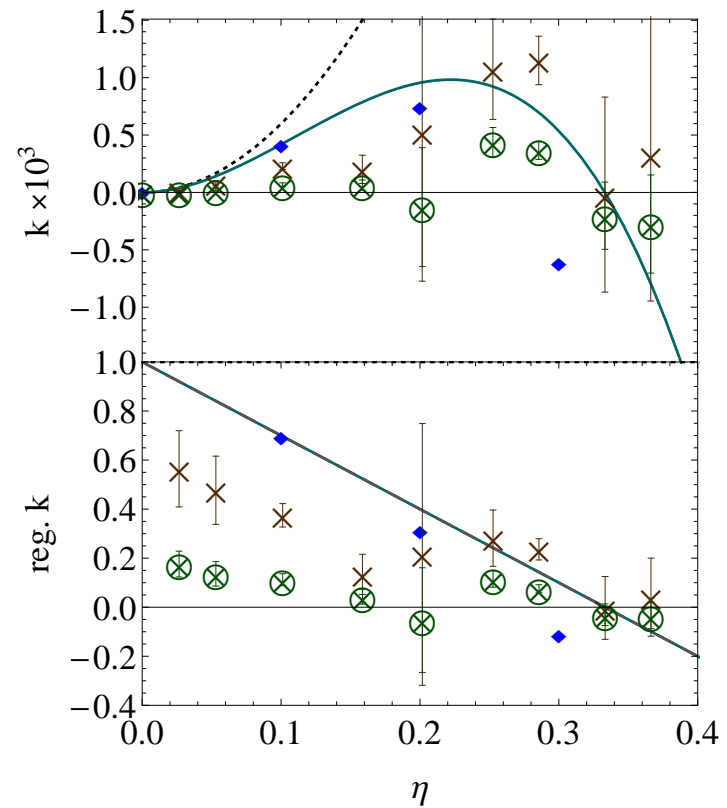

Figure 6. (a) Bending constant $k$ for both d-RR and e-RR (in units of $k_{\mathrm{B}} T$ ). (b) Magnitude plotted in (a) but in its regularized form. Squares (blue) are FMT results from Blokhuis [4], crosses and circles with crosses are obtained from MD results by Laird et al.[3] Continuous line (lightblue) is the proposed expression while dotted line is the exact series expansion truncated at the last known term.

d-RR and e-RR, which should be identical. In Fig. 6 (a) the scale in the ordinate axes shows that $k$ is a very small magnitude. There, one can observe a large spreading of the data. Moreover, the MD results show some degree of inconsistency in the values obtained adopting the d-RR (crosses) and the e-RR (circles with crosses) while largest error bars covers the complete range of variation of $k$ with $\eta$. All these features might indicate that the $\mathrm{MD}+\mathrm{fit}$ procedure used to extract the $k$ values is not completely confidence. In addition, the three FMT values suggest a nearly cubical behavior with a root at $\eta \sim 0.28$. The FMT approach is free from fitting uncertainties and has a high degree of self-consistence that enables to estimate absolute errors that are very smalls, eg. $\Delta k \simeq 10^{-8}$ for $\eta=0.1$. However, a critical revision of the FMT adopted in Ref. [4] signals that the values for $k$ may be biased by the inaccuracy of the FMT itself at the high degree of detail shown in Fig. 66 (a) where the diameter of the circles is $2 \times 10^{-4}$ (in $k_{B} T$ units). This makes unclear the confidence that one should assign to these results to describe by themselves the subtle behavior of $k(\eta)$ for the true HS system. In particular, one source of inaccuracy in the adopted FMT is the use of the Percus-Yevick pressure EOS [23] that fails with increasing $\eta$. Based on Fig. 6 (b) one note that FMT results suggest an overall linear behavior for the regularized form of $k$. Turning to MD results, at low density it is clear that none of both sets of data (that correspond 


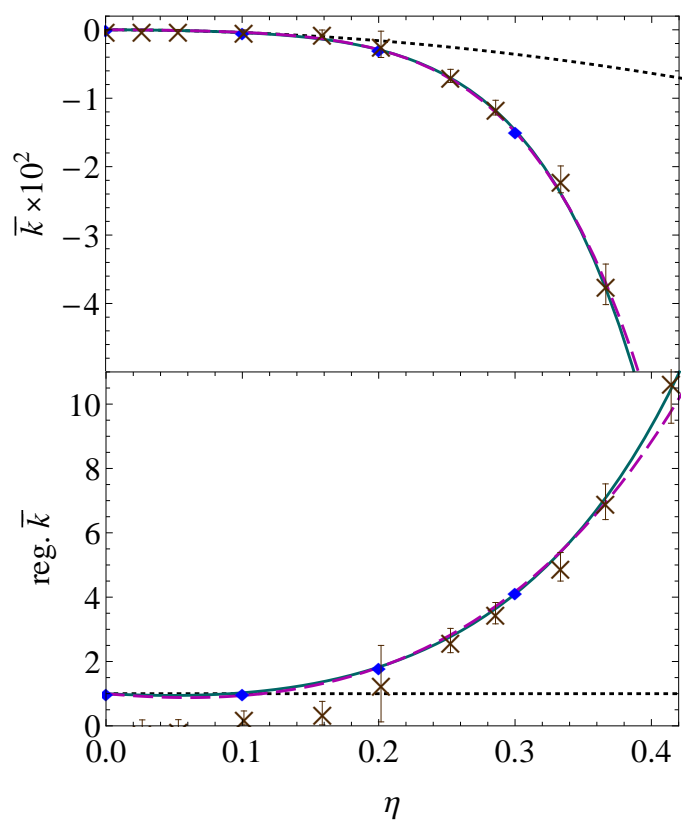

Figure 7. (a) Bending constant $\bar{k}$ (in units of $k_{\mathrm{B}} T$ ) in the framework of d-RR. (b) Magnitude plotted in (a) but in its regularized form. Lines and symbols were described in Fig. 6. except dashed line (magenta) obtained from Eqs. (30, 31) as $c k-2 k$.

to $\mathrm{d}-\mathrm{RR}$ and e-RR) point to the correct limiting value reg. $k \rightarrow 1$ with $\eta \rightarrow 0$, and also, that the degree of inconsistency between both make them non-confidence at low $\eta$. On the other hand, for $\eta \gtrsim 0.22$ the decrease of $k(\eta)$ and its nearly zero value is well established by the approximate coincidence of MD and FMT results. Thus, I do not consider the MD results for $\eta \lesssim 0.22$, but I assume a simple linear behavior of regularized $k$ and make a crude estimate of the slope by considering the FMT value at the smaller density $(\eta=0.1)$ and the MD results for $\eta \gtrsim 0.22$. Based on this analysis, I found a slope of -3 which corresponds to Eq. (31) and is plotted in the figure. For the Gaussian-curvature rigidity I propose

$$
\beta \bar{k}=-\frac{\eta^{2}}{8 \pi} \frac{1-4.2(1-6 \eta) \eta}{(1-\eta)^{2}}, \quad(\mathrm{~d}-\mathrm{RR})
$$

where -4.2 was found by fitting. In Fig 7 (a) it is plotted $\bar{k}$ in d-RR. There, one can verify the accurate fitting of Eq. (32) to MD and FMT data, as well as, the consistence between the fitted $\bar{k}$ and that found from Eqs. (30, 31) as $c k-2 k$. In Fig 7 (b) is apparent that for $\eta \lesssim$ $0.2 \mathrm{MD}$ data does not has the correct behavior because it does not go to unit when $\eta \rightarrow 0$, this inconsistency is clearly related to that found in Fig. 6 (b) and makes that MD data for this range of $\eta$ be non-confidence. Therefore, Eq. (32) is obtained by fitting the three FMT points and those of MD for $\eta \gtrsim 0.2$. The consistence of the approach is verified by the coincidence with the alternative route to $\bar{k}$ plotted in dashed line. The expression in Eq. (32)

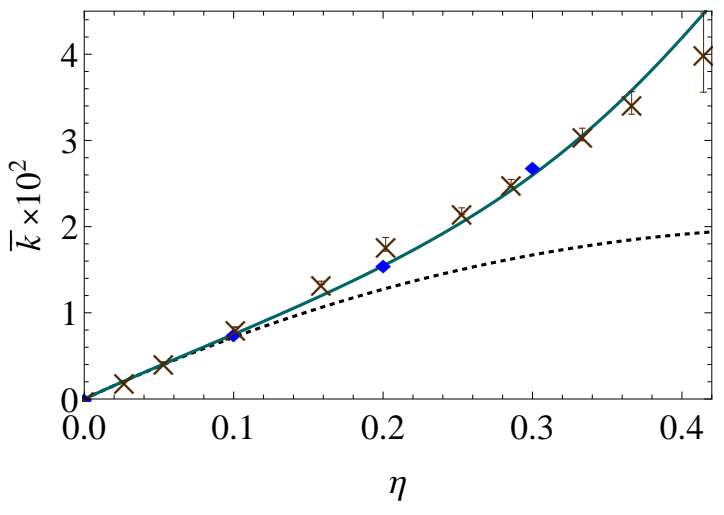

Figure 8. Bending constant $\bar{k}$ (in units of $k_{\mathrm{B}} T$ ) in the framework of e-RR. Lines and symbols were described in Fig. 6 .

transformed to the e-RR is plotted in Fig. 8 Again, the curve fits very well the data which validates the obtained description.

\section{FINAL REMARKS}

In this work exact expressions were derived for the Tolman length and for the combination of curvature terms $2 k+\bar{k}$ up to order three in the packing fraction. Furthermore, the dependence of both the bending rigidity and the rigidity constant associated with the Gaussian curvature were found up to order two in the packing fraction. All these findings are absolute in the sense that they do not imply the assumption of non-exact EOS and can be readily transformed on exact grounds to any reference region. Moreover, the functional dependence of these curvature-thermodynamic properties with the packing fraction away from $\eta \gtrsim 0$ was established on the basis of an approximate and accurate fitting procedure.

Based on the truncated power series in the packing fraction, it were presented definitive evidence showing that for the HS fluid in contact with hard-curved walls $k \neq 0$, and then, that Eq. (2) proposed by König et al. [8, 9] based on Hadwiger theorem [6, 24] is not a complete expression for $\gamma(J, K)$, at least for the studied system. The same conclusion extends to the complete morphological thermodynamic approach which might be considered a good approximate theory but not an exact one. This result is in good agreement with that found previously using FMT [4]. Even that, it must be remarked that the use of free energy density functional theories like FMT for the evaluation of small and sensible quantities should be done with caution. FMT is an approximate theory for inhomogeneous fluids, and thus, it is a priori unclear which are the boundaries of reliability of the involved approximations. Particularly, its capability to describe the subtle behavior of $k$ and its degree of confidence should be further studied.

Moreover, it is shown that the order $O\left(A R^{-4}\right)$ terms in $\gamma(R)$ are non-null and thus, that the truncation up 
to order $J^{2}$ and $K$ of the Helfrich expansion is also incomplete and does not enable to accurately describe the known properties of the HS inhomogeneous system. Notably, the expressions for $\tau_{2(c y l)}(R)$ and $\tau_{3(s p h)}(R)$, that in this work were truncated to order $A / R^{3}$, enables to readily extend the results to any order in powers of $R^{-1}$ showing that the Helfrich expression is also approximate if one truncate it to any finite order in $R^{-1}$. The accuracy of the analytic expressions for $\gamma, \delta \gamma, c k, k$ and $\bar{k}$ found by fitting is largely restricted by the small discrepancies between the different theoretical methods utilized to obtain their values. In this sense, the development of a direct MonteCarlo-based method to evaluate the curvature-thermodynamic properties might be necessary to find more reliable and accurate results.

\section{ACKNOWLEDGMENTS}

I am grateful to Jung Ho Yang and David A. Kofke for kindly providing me with the surface tension MonteCarlo data and to Gabriela Castelletti and Claudio Pastorino for helpful discussions and comments. This work was supported by Argentina Grants CONICET PIP-0546/10, UBACyT 20020100200156 and ANPCyT PICT-2011-1887.
[1] J. H. Yang, A. J. Schultz, J. R. $\begin{array}{llll}\text { Errington, and } & \text { D. A. Kofke, } \\ \text { The Journal of Chemical Physics 138, } & 134706 & \text { (2013) }\end{array}$

[2] B. B. Laird and R. L. Davidchack, The Journal of Chemical Physics 132, 204101 (2010)

[3] B. B. Laird, A. Hunter, and R. L. Davidchack, Phys. Rev. E 86, 060602 (2012).

[4] E. M. Blokhuis, Phys. Rev. E 87, 022401 (2013)

[5] W. Helfrich, Zeitschrift für Naturforschung. Teil C: Biochemie, Biophysik, Biologie, Virologie 28, 693 (1973).

[6] H. Hadwiger, Vorlesungen über Inhalt, Oberfläche und Isoper (Springer, Basel, 1957).

[7] P. Bryk, R. Roth, K. R. Mecke, and S. Dietrich, Phys. Rev. E 68, 031602 (2003).

[8] P. M. König, R. Roth, and K. R. Mecke, Phys. Rev. Lett. 93, 160601 (2004).

[9] P. M. König, P. Bryk, K. R. Mecke, and R. Roth, Europhysics Letters 69, 832 (2005).

[10] T. L. Hill, Statistical Mechanics (Dover, New York, 1956).

[11] A. Bellemans, Physica 28, 493 (1962)

[12] S. Sokołowski and J. Stecki, Acta Physica Polonica 55, 611 (1979).
[13] I. Urrutia, Journal of Statistical Physics 131, 597 (2008)

[14] I. Urrutia, The Journal of Chemical Physics 135, 024511 (2011), erratum: ibid. 135(9), 099903 (2011).

[15] I. Urrutia, The Journal of Chemical Physics 133, 104503 (2010)

[16] The coefficient $b_{4}$ is known exactly being its value $-\pi^{2}(876 \sqrt{2}+94243 \pi+8262 \operatorname{ArcCsc} 3) / 90720$ while $b_{5}$ is evaluated using data of Ref. [17]. Both, $a_{4}$ and $a_{5}$ are evaluated using $a_{2}, a_{3}$ and data taken from Ref. [1]..

[17] N. Clisby and B. M. McCoy, Journal of Statistical Physics 122, 15 (2006).

itrotte Reiss, H. L. Frisch, E. Helfand, and J. L. Lebowitz, The Journal of Chemical Physics 32, 119 (1960).

[19] H. Hansen-Goos and R. Roth, Journal of Physics: Condensed Matter 18, 8413 (2006)

[20] J. Kolafa, S. Labík, and A. Malijevský, Phys. Chem. Chem. Phys. 6, 2335 (2004).

[21] D. Henderson and M. Plischke, Proceedings of the Royal Society of London. A. Mathematical and P

[22] MonteCarlo simulation results for $\gamma$ previously published in Ref. 1] were kindly provided by Jung Ho Yang and David A. Kofke.

[23] M. S. Wertheim, Phys. Rev. Lett. 10, 321 (1963).

[24] K. R. Mecke, International Journal of Modern Physics B 12, 861 (19) 